\title{
Visuo-tactile integration and body ownership during self-generated action
}

\author{
G. Rognini, ${ }^{1,2,3}$ A. Sengül, ${ }^{1,2,3}$ J. E. Aspell, ${ }^{2}$ R. Salomon, ${ }^{1,2}$ H. Bleuler ${ }^{3}$ and O. Blanke ${ }^{1,2,4}$ \\ ${ }^{1}$ Center for Neuroprosthetics, Ecole Polytechnique Fédérale de Lausanne, Lausanne, Switzerland \\ ${ }^{2}$ Laboratory of Cognitive Neuroscience, Brain Mind Institute, Ecole Polytechnique Fédérale de Lausanne, Lausanne, Switzerland \\ ${ }^{3}$ Laboratory of Robotic Systems, School of Engineering, Ecole Polytechnique Fédérale de Lausanne, Lausanne, Switzerland \\ ${ }^{4}$ Department of Neurology, University Hospital Geneva, Geneva, Switzerland
}

Keywords: agency, cross-modal congruency effect, internal model, movement, multisensory

\begin{abstract}
Although there is increasing knowledge about how visual and tactile cues from the hands are integrated, little is known about how self-generated hand movements affect such multisensory integration. Visuo-tactile integration often occurs under highly dynamic conditions requiring sensorimotor updating. Here, we quantified visuo-tactile integration by measuring cross-modal congruency effects (CCEs) in different bimanual hand movement conditions with the use of a robotic platform. We found that classical CCEs also occurred during bimanual self-generated hand movements, and that such movements lowered the magnitude of visuo-tactile CCEs as compared to static conditions. Visuo-tactile integration, body ownership and the sense of agency were decreased by adding a temporal visuo-motor delay between hand movements and visual feedback. These data show that visual stimuli interfere less with the perception of tactile stimuli during movement than during static conditions, especially when decoupled from predictive motor information. The results suggest that current models of visuo-tactile integration need to be extended to account for multisensory integration in dynamic conditions.
\end{abstract}

\section{Introduction}

Multisensory integration is considered to be the basis for important aspects of bodily self-consciousness, notably body ownership and the sense of agency (Haggard et al., 2003; Jeannerod, 2007). Recently, the cross-modal congruency effect (CCE) has been proposed as a measure of visuo-tactile spatial integration and hand ownership (Pavani et al., 2000; Zopf et al., 2010). In the crossmodal congruency task, participants are asked to respond to the elevation of tactile stimuli, and they typically perform worse when a distracting visual stimulus occurs at an incongruent elevation with respect to the tactile target. The CCE [reaction time (RT)-error incongruent minus RT-error congruent] is typically larger when the visual and tactile stimuli are closer to each other in space (Spence et al., 2004a).

Although visuo-tactile integration and body ownership generally occur under dynamic conditions in which constant updating of sensory and motor cues is required (Klatzky et al., 2008), this topic has received scant attention. This can probably be attributed to the technical difficulties in creating conflicts between touch and vision during active movements. Experimental evidence supports the existence of an internal model that, making it possible to monitor self-gener-

Correspondence: O. Blanke, ${ }^{2}$ Laboratory of Cognitive Neuroscience, as above. E-mail: olaf.blanke@epfl.ch

Received 19 October 2011, revised 10 December 2012, accepted 13 December 2012 ated actions, influences perceptual judgements (Wolpert et al., 1995; Blakemore et al., 1999). For example, self-generated actions have been shown to attenuate the perception of somatosensory stimuli (Collins et al., 1998; Bays et al., 2006). However, in the visual domain, although moving stimuli are generally more difficult to perceive than static ones (Jacobs, 2002; Triesch et al., 2002), the visual consequences of self-generated movements are easier to perceive (Tsakiris et al., 2005; Salomon et al., 2011). Another important aspect of self-generated actions is the sense of agency, i.e. the feeling of control over one's actions (Tsakiris \& Haggard, 2005; Salomon et al., 2009). This has been proposed to rely on the match between the motor signals generating the movement and the corresponding sensory feedback and predicted feedback signals [see Frith (2005) for a review]. As self-generated movement influences both tactile and visual perception, its effects on visuo-tactile integration are especially important.

Here, we used a robotic and virtual reality (VR) platform to combine the lines of research mentioned above. We first showed that CCEs could be elicited in our robotic platform setup. Second, we hypothesised that, if self-generated movement attenuates tactile perception, we should find higher CCEs than in the static condition. However, if the movement rendered the visual information regarding the location of the visual distractors less reliable, this would lead to a reduction in visual interference. Thus, we would expect a smaller CCE magnitude in the movement condition than in the static condition. Third, we tested whether the results of study 2 were related to 
predictive information from self-generated movements. This was done by inserting an additional mismatch between the generated motor signals and the corresponding visual signals. We expected a larger CCE magnitude in the synchronous condition than in the asynchronous condition, as tactile cues would be perceived to be closer to the visual distractors during the synchronous condition (Pavani et al., 2000; Aspell et al., 2009).

\section{Materials and methods}

\section{Subjects}

A total of 30 healthy right-handed participants took part in these experiments: 11 (two females; mean age, 28 years), in study $1 ; 12$ (five of whom also participated in study 1; four females; mean age, 27 years) in study 2; and 12 (five females; mean age, 25 years) in study 3. All participants had normal or corrected to normal vision, and had no history of neurological or psychiatric conditions. All participants gave written informed consent, and were compensated for their participation. The study protocol was approved by the local ethics research committee - La Commission d'éthique de la recherche Clinique de la Faculté de Biologieet de Médecine - at the University of Lausanne, Switzerland, and was performed in accordance with the ethical standards laid down in the Declaration of Helsinki.

\section{Materials}

We employed a robotic system - a bimanual haptic interface that has been designed as a training device for users of the da Vinci system (Intuitive Surgical, Sunnyvale, CA, USA). The da Vinci system is a robotic device that is used worldwide for different surgical procedures with a minimally invasive approach (Ayav et al., 2004; Lanfranco et al., 2004). The employed haptic interface provides seven degrees of freedom in motion for each hand and force feedback in three translations (Kenney et al., 2009; Lerner et al., 2010).
The force feedback and the tracking of the hand movements are provided through a cable-driven system (cables connect the two grippers to the motors). This results in grippers with low weight and inertia, and allows for transparent human-machine interactions, making it possible to simulate a wide range of bimanual manipulations. In order to measure CCEs (the performance difference between incongruent and congruent visuo-tactile conditions: RT-error incongruent minus RT-error congruent), we used four small vibrating motors [Precision Micro Drives shaftless vibration motors, model 312-101, $3 \mathrm{~V}, 80 \mathrm{~mA}, 9000$ r.p.m. (150 Hz), 1.7 g]. The motors had a diameter of $12 \mathrm{~mm}$ and a length of $3.4 \mathrm{~mm}$, and reached maximal rotation velocity in approximately $50 \mathrm{~ms}$. Motors were attached to the skin with tape. When activated, these vibrotactile stimulators gave rise to a clearly perceptible and easily localisable supra-threshold vibrotactile sensation. The two grippers of the haptic device were connected through a frame in order to maintain a constant distance between the hands during the different experiments and to give a more realistic feeling of holding a cube. The distances between the hands and between the thumbs and the fingers were $20 \mathrm{~cm}$ and $8 \mathrm{~cm}$, respectively. No mass was simulated, and participants felt only the weight (approximately $100 \mathrm{~g}$ ) of the mechanical frame $(24 \times 8 \mathrm{~cm})$ used to constrain the distance between the hands.

Participants sat at a table and held the two grippers (Fig. 1). The index and thumb of both hands were positioned in the haptic device, and their movements and interactions with virtual objects were presented in two dimensions on a head-mounted display (HMD) in real time.

The scale factor - the ratio between a distance in the virtual world and the physical world - was 1.5. This was chosen to compensate for the typical underestimation of perceived distance that occurs in VR when HMDs are used, and therefore to provide more realistic interactions (Loomis \& Knapp, 2003). Foam and rubber padding was used to insulate vibrotactile stimulators from the surrounding material, thus minimising any conduction of vibrations through the haptic device itself. Vibrotactile stimuli were driven by
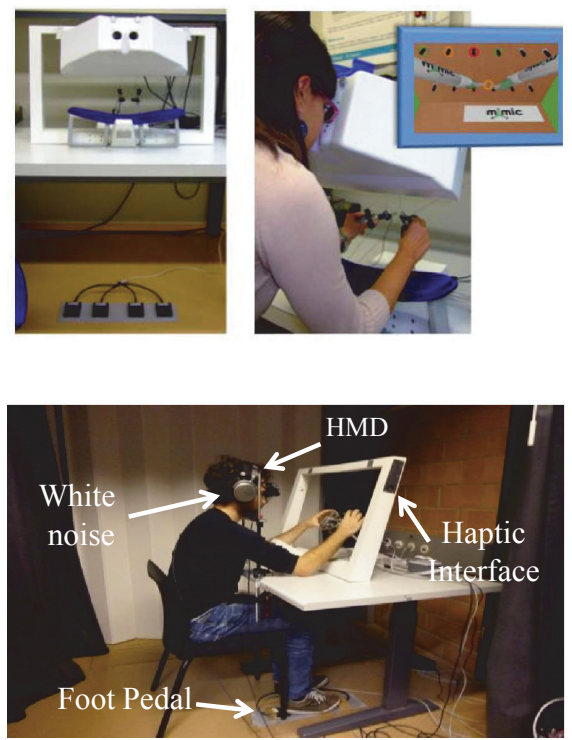

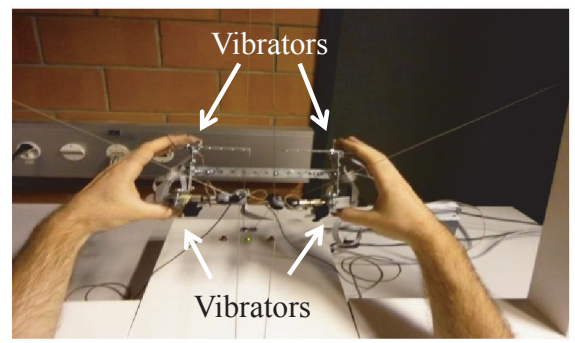

Distractors

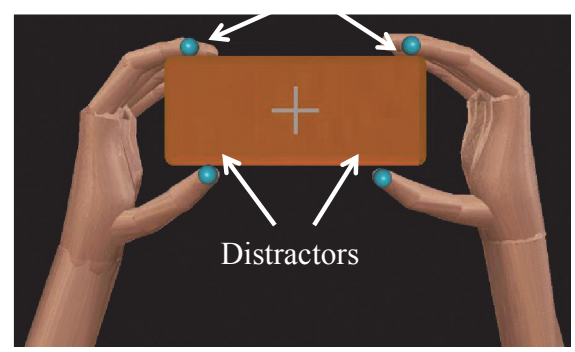

FIG. 1. Experimental setup. Left panel, upper row: prototype of the haptic interface used in the present study (surgical training interface). Left panel, bottom row: participants sat at a table and held the structure connected to the two grippers of the haptic device. The index and thumb of both hands were positioned in the haptic device. Movements, virtual object and virtual hands were presented on a visual display in real time. Right panel, upper row: participants were asked to hold the frame connecting the two grippers of the haptic device. Right panel, bottom row: virtual scene - two virtual hands holding a block with a fixation cross in the middle. The four dots show the positions of the visual distractors. During the experiment, distractors were presented only one at time (see also Materials and methods). 
an electrical signal generated by two data acquisition cards (NI PCI6014, 16-bit resolution, $250 \mathrm{kS} / \mathrm{s}, 2$ analog output, 8 digital i/o 2 24-bit counters; NI PCI 6052E, 18-bit resolution, $625 \mathrm{kS} / \mathrm{s}, 2$ analog output, 8 digital i/o 2 24-bit counters) installed in a desktop computer (Intel Core i7 CPU with $2.8 \mathrm{GHz}, 3 \mathrm{~GB}$ of RAM, Graphic Card NVIDIA GeForce 9800 GT). To minimise any unwanted reflections, the participants were seated in a dimly illuminated room.

An open source platform (CHAI 3D; http://www.chai3d.org) and a set of $\mathrm{C}++$ libraries were used for the modeling and visualisation of the virtual world (Wei et al., 2008). This platform supports several commercial haptic devices, and it is possible to extend it to support new customised force feedback devices. We extended this platform for the present experiments by adding drivers and libraries from our customised force feedback device. A virtual world showing two hands holding a block was projected in real time (except for the asynchronous blocks; see below) onto an HMD (eMagin Z800 3DVisor, approximately $40^{\circ}$ angle view, 1.44-megapixel resolution, $50 \mathrm{~cd} / \mathrm{m}^{2}$ brightness, $227 \mathrm{~g}$ ). A fixation cross was presented in the middle of the display, and a chinrest was used to constrain the participant's head position. To record the participant's responses, two pedals were attached to the floor next to the participant's right foot. The distance between the two pedals was adjusted to fit the participant's foot size. One of the pedals was placed under the heel and the other under the toe of the participant's right foot. These adaptations were made on the basis of previous work on visuo-tactile CCEs (i.e. Spence et al., 2004a). White noise was presented over headphones to mask the sound of the vibrotactile stimulators and the operation of other hardware during the experiments. The maximum latency related to information transfer delays and computational processing during movements of the physical hands (of the participants) and virtual hands (on the display) was equal to $20 \mathrm{~ms}$ (sampling rate of $50 \mathrm{~Hz}$, delay for the near-synchronous condition in experiments using movement; see below). However, the end-toend latency could have been affected by unmeasured additional delays introduced by the peripheral devices (Di Luca, 2010). Nevertheless, these delays were not visually perceivable, suggesting that their latency was brief.

\section{Procedure}

As in previous CCE studies (Pavani et al., 2000; Spence et al., 2004a; Shore et al., 2006; Aspell et al., 2009; Zopf et al., 2010), participants were asked to make rapid elevation discrimination responses (by raising the toes or the heel of the right foot positioned on the foot pedal) to vibrotactile targets presented either at the 'top' (to one of their index fingers) or at the 'bottom' (to one of their thumbs). The visual cues had a duration of $100 \mathrm{~ms}$, and preceded the vibrotactile stimulus by $100 \mathrm{~ms}$. This stimulus onset asynchrony was chosen on the basis of results of a previous study showing that this timing boosts CCE magnitude (Shore et al., 2006). Vibrotactile cues had a duration of $30 \mathrm{~ms}$.

Study 1 was designed to investigate whether CCEs can be observed in the present robotic setup. Thus, the participants were asked not to move their hands (as in most previous CCE studies) (Pavani et al., 2000; Spence et al., 2004a; Shore et al., 2006; Aspell et al., 2009; Zopf et al., 2010). For studies 2 and 3, we also recorded the positions of the participants' right and left hands (an example is shown in Fig. 2). The movement profile was calculated with respect to the left arm, and as only three translations were allowed, the different trajectories for the left arm, the center of mass and the right arm only differed by an offset. Considering the left hand trajectory as the reference, the trajectory of the center of mass

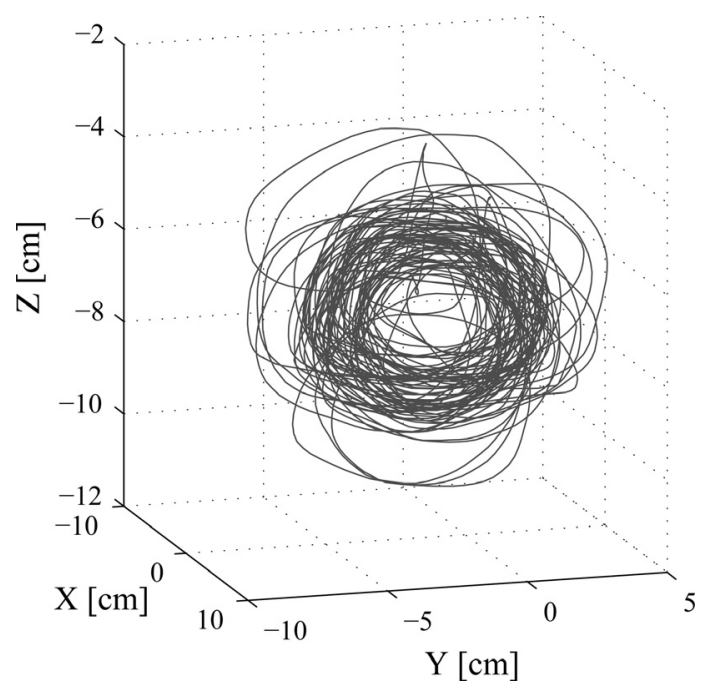

FIG. 2. Trajectories. Example of three-dimensional trajectories from one participant in study 2 . In study 2, the average trajectory norm was $5.1 \mathrm{~cm}$ (SE, $0.2 \mathrm{~cm}$ ) and the average velocity was $3.2 \mathrm{~cm} / \mathrm{s}(\mathrm{SE}, 0.5 \mathrm{~cm} / \mathrm{s})$, whereas in study 3 the average trajectory norm was $13.3 \mathrm{~cm}(\mathrm{SE}, 0.7 \mathrm{~cm})$ and the average velocity was $6.0 \mathrm{~cm} / \mathrm{s}(\mathrm{SE}, 0.6 \mathrm{~cm} / \mathrm{s})$.

was shifted by $10 \mathrm{~cm}$ rightwards and the right hand trajectory by $20 \mathrm{~cm}$. As mentioned previously, these distances were kept fixed by a mechanical frame. In both study 2 (only during the movement condition) and study 3, participants were asked to perform repeated slow clockwise circular movements in the frontal plane, while keeping their elbows on the table. The latter was done to limit their movement range. As the haptic interface is able to track only three translations, participants were also asked to avoid rotating their wrists. We decided to have our participants perform circular movements in the frontal plane, because front to back movements, for instance, would have caused differences in scaling of the virtual hand size, the object, and the visual distractors (all of which are smaller when further away). Moreover, as the relative weighting of vision and proprioception has been shown to depend on depth (van Beers et al., 2002), keeping the movement in the frontal plane (at least approximately) allowed us to avoid any possible bias resulting from different responses given at different depths. In all of the experiments, participants performed a training session (range, two or three blocks of 16 trials) before starting the study [similarly to Pavani et al. (2000)]. They were considered to be able to start as soon as their performances reached $85 \%$ accuracy across all of the trials. This was done to ensure that participants were able to recognise the tactile stimuli. In study 2, the training was for both the static and the movement conditions, and was counterbalanced across participants. In study 3, participants were trained in the synchronous condition. During the training phase, participants were corrected by the experimenter if they were considered to move too fast, too move to slow, or to have too large a range of movement. In study 3 , the delay chosen for the asynchronous condition was $600 \mathrm{~ms}$, as previous studies on ownership and agency found strong effects within this delay range (Tsakiris et al., 2006). Concerning the number of blocks, in study 1 there were 24 blocks per condition, and in studies 2 and 3 there were 16 blocks. Each block was composed of 16 trials. The order of blocks was always counterbalanced across participants. In study 3, participants also completed a short questionnaire including items on agency for the seen movement and ownership of the seen hands, with a seven-point Likert scale (1, strongly disagree; 4, not certain; 7, strongly agree), at the end of the experiment. The 
TABLE 1. Agency and ownership questionnaire: statements included in the questionnaire completed by participants

During the experiment there were times when:

Q1: I felt as if the virtual hands were my hands.

Q2: I felt as if the movement of the virtual hands was my movement.

Q3: It seemed as if I had lights on my hands.

Q4: I felt as if my hands were turning 'virtual'.

questions were adapted from previous related work by Pavani et al. (2000) (Table 1).

\section{Data analysis}

For CCE analysis, only trials with correct responses and with RTs between $200 \mathrm{~ms}$ and $3000 \mathrm{~ms}$ were considered (following a previous study on tool use and a cross-modal congruency task) (Holmes et al., 2007a). Statistical outliers were removed per participant per condition, following the modified recursive procedure reported by Van Selst \& Jolicoeur (1994). Removed trials accounted for $7.3 \%$ of trials in study 1 . In study $2,4.2 \%$ of trials were removed for the static condition and $6.9 \%$ for the movement condition, whereas in study $3,8.1 \%$ of trials were removed for the synchronous condition and $8.6 \%$ for the asynchronous condition.

For movement analysis, raw movement data were processed in order to extract two parameters: average velocity and trajectory norm. Velocity and trajectory norm - the latter defined as the Euclidian norm of the three-dimensional trajectory between trials were calculated as an average over trials. This was done to assess whether participants respected the instruction to move in a circular clockwise way, to assess whether motor performances (velocity and motion range) differed between conditions, and, in general, to investigate possible parallels between the motor and the perceptual performances (Brozzoli et al., 2009, 2010). Raw movement data from all the participants were also processed, in order to calculate the standard deviation of position for each of the three axes. This was done to provide a measure of how much participants moved during the different conditions.

\section{Statistical analysis}

The experimental factors in study 1 were congruency (congruent and incongruent) and side (same and different). For study 2, the factors were congruency (congruent and incongruent), side (same and different), and movement (static and movement). For study 3, the factors were congruency (congruent and incongruent), side (same and different), and synchrony (synchronous and asynchronous). RTs and errors were found to have equal variances (Mauchly's test for sphericity), and were analysed with two-tailed repeated-measures ANOvAs. The Newman-Keuls test and two-tailed $t$-tests were used for post hoc analysis. Velocity and trajectory workspace for studies 2 and 3 were found to have equal variances (Mauchly's test for sphericity), and were analysed with two-tailed repeated-measures ANOVAs with the same factorial designed as employed for the RTs and errors.

For statistical analysis of the questionnaire ratings in study 3 , we used Wilcoxon matched-pairs test. All numerical values represent the mean \pm between-subjects standard error of the mean. Significant effects were reported for $P \leq 0.05$. The discussion of the results focuses on RT data rather than errors, as these have been shown to be more sensitive for the CCE (Pavani et al., 2000; Austen et al., 2004; Shore et al., 2006; Aspell et al., 2009).

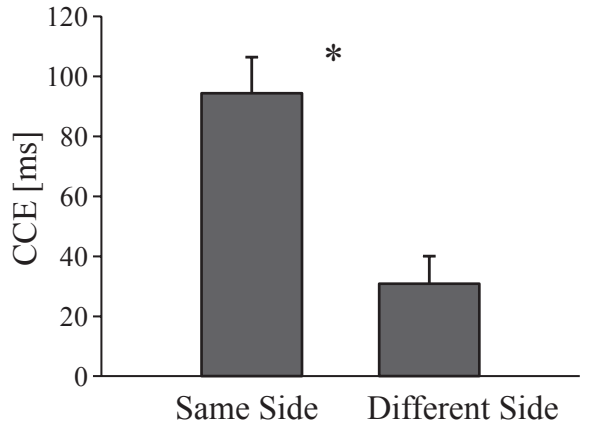

FIG. 3. Robotically mediated visuo-tactile integration (study 1). Mean CCEs are shown for RTs. Values are RT in incongruent trials minus RT in congruent trials. Error bars show standard errors of the mean. Note the stronger CCE if visual distractors are presented on the same side as the tactile stimulus.

\section{Results}

\section{Study 1 - CCE magnitude in a robotic setup}

\section{CCE analysis}

RTs across the four experimental conditions ranged between 436 and $875 \mathrm{~ms}$. As expected on the basis of previous work, the CCE magnitude was larger in the same-side conditions than in the different-side conditions, by $69 \mathrm{~ms}$ [standard error (SE), $6 \mathrm{~ms}$ ]. The ANOVA on RTs revealed a main effect of congruency $\left(F_{1,10}=40.52\right.$, $P<0.00001)$ and a significant interaction between side and congruency $\left(F_{1,10}=117.73, P<0.00001\right)$. CCEs were significantly larger in the same-side conditions than in the different-side conditions $\left(t_{1,10}=10.85, P<0.00001\right.$; Fig. 3 and Table 2).

TABLE 2. RT and error results: mean RT and percentage of errors for tactile targets in studies $1-3$ as a function of the visual distractor with respect to the target, the distractor's congruence with the target, and the experimental condition (standard errors are in parentheses)

\begin{tabular}{|c|c|c|c|}
\hline Target-distractor congruence & $\begin{array}{l}\text { Position of } \\
\text { distractor }\end{array}$ & $\begin{array}{l}\text { Reaction } \\
\text { time (ms) }\end{array}$ & Error $(\%)$ \\
\hline \multicolumn{4}{|l|}{ Study 1} \\
\hline \multirow[t]{2}{*}{ Congruent } & Same & $592(31)$ & $4.5(1.0)$ \\
\hline & Different & $626(31)$ & $5.4(1.5)$ \\
\hline \multirow[t]{2}{*}{ Incongruent } & Same & $695(27)$ & $7.3(1.7)$ \\
\hline & Different & $659(25)$ & $4.3(0.9)$ \\
\hline \multicolumn{4}{|l|}{ Study $2-$ Static } \\
\hline \multirow[t]{2}{*}{ Congruent } & Same & $546(25)$ & $1.9(0.5)$ \\
\hline & Different & $583(28)$ & $2.3(0.8)$ \\
\hline \multirow[t]{2}{*}{ Incongruent } & Same & $636(36)$ & $4.7(1.5)$ \\
\hline & Different & $614(34)$ & $2.5(0.7)$ \\
\hline \multicolumn{4}{|l|}{ Study 2 - Movement } \\
\hline \multirow[t]{2}{*}{ Congruent } & Same & $570(29)$ & $5.4(1.5)$ \\
\hline & Different & $599(28)$ & $3.6(1.0)$ \\
\hline \multirow[t]{2}{*}{ Incongruent } & Same & $643(35)$ & $7.1(1.7)$ \\
\hline & Different & $614(33)$ & $4.7(1.0)$ \\
\hline \multicolumn{4}{|l|}{ Study 3 - Synchronous } \\
\hline \multirow[t]{2}{*}{ Congruent } & Same & $593(18)$ & $5.1(1.3)$ \\
\hline & Different & $624(20)$ & $4.1(0.7)$ \\
\hline \multirow[t]{2}{*}{ Incongruent } & Same & $700(27)$ & $6.3(1.0)$ \\
\hline & Different & $662(21)$ & $5.7(1.0)$ \\
\hline \multicolumn{4}{|l|}{ Study 3 - Asynchronous } \\
\hline \multirow[t]{2}{*}{ Congruent } & Same & $618(22)$ & $5.2(1.0)$ \\
\hline & Different & $635(25)$ & $4.1(0.6)$ \\
\hline \multirow[t]{2}{*}{ Incongruent } & Same & 705 (29) & $8.3(1.6)$ \\
\hline & Different & $680(26)$ & $6.2(1.4)$ \\
\hline
\end{tabular}


The ANOVA on errors revealed a similar tendency: a significant interaction between side and congruency $\left(F_{1,10}=6.451, P<0.03\right)$, and a tendency towards significance for congruency $\left(F_{1,10}=4.078\right.$, $P=0.07$ ). CCEs (incongruent errors minus congruent errors) were significantly larger in the same-side conditions than in the differentside conditions $\left(t_{1,10}=2.53, P<0.03\right.$; Table 2$)$. A comparison between the ANOVA on RTs and the ANOVA on errors revealed the same tendency for the significant effects (congruency and interaction between side and congruency), and thus provide no evidence for speed-accuracy tradeoffs in the present data.

The data of study 1 show that, in the present robotic setup, the magnitudes of general RTs and error rates, as well as the respective CCEs, were of similar magnitude as found in previous standard setups (e.g. Spence et al., 2004a).

\section{Study 2 - CCE magnitude during hand movements}

\section{CCE analysis}

RTs across the eight experimental conditions ranged between $412 \mathrm{~ms}$ and $843 \mathrm{~ms}$. The CCE magnitude was found to be larger in the sameside conditions than in the different-side conditions, and also reflected movement. The ANOVA on RTs revealed a main effect of congruency $\left(F_{1,11}=31.75, P<0.001\right)$ and a significant interaction between side and congruency $\left(F_{1,11}=74.20, P<0.0001\right)$, confirming that CCEs were significantly larger in the same-side conditions than in the different-side conditions $\left(t_{1,11}=7.11, P<0.0001\right)$.

Crucially, the analysis revealed a significant interaction between movement and congruency $\left(F_{1,11}=7.97, P<0.02\right)$. A NeumanKeuls post hoc analysis revealed that the significant interaction between movement and congruency was caused by a significant difference between the static congruent condition and the movement congruent condition $(P<0.0002)$, which was absent between the incongruent conditions $(P>0.35)$ (Fig. 4). Further analysis revealed that CCEs in the static conditions were significantly larger than in the movement conditions $\left[t_{1,11}=2.82, P<0.02\right.$; Fig. 4 (inset) and
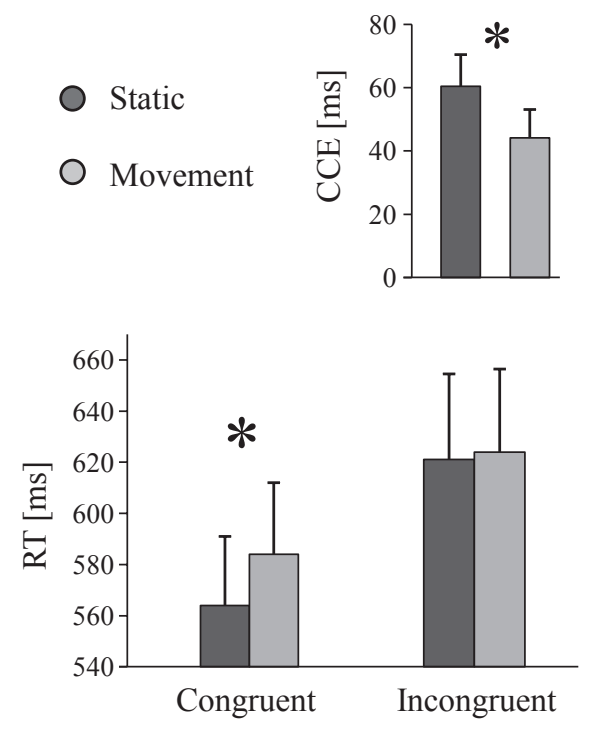

FIG. 4. Movement decreases visuo-tactile integration (study 2). Average RTs are shown, revealing the interaction between movement and congruency. Inset: mean CCEs (RTs) are shown in the static and the movement conditions. Error bars show the standard error of the mean. See results for study 2. Note the weaker CCE during the movement condition.
Table 2]. Results at the level of the single subject showed larger RTs in the movement congruent condition than in the static congruent condition in nine of 12 subjects (Fig. S1). Of these nine subjects, only three showed a very small difference $(<10 \mathrm{~ms})$. The three subjects showing a difference in the opposite direction (static congruent larger than movement congruent) showed only small differences $(12,10$ and $17 \mathrm{~ms})$. We therefore conclude that results at the level of the single subject are consistent with those at the group level.

The ANOVA on errors revealed a main effect of movement $\left(F_{1,11}=11.22, P<0.01\right)$, a main effect of side $\left(F_{1,11}=6.56\right.$, $P<0.03)$, and a tendency towards significance for a main effect of congruency $\left(F_{1,11}=4.24, P=0.064\right)$. There were no significant interactions (Table 2). Comparisons between the ANOvA on RTs and the ANOVA on errors revealed the same tendency for the common significant effect of congruency, suggesting that there were no speed -accuracy tradeoffs.

\section{Trajectory analysis}

In study 2, movement analysis revealed that the average (betweensubjects) trajectory norm was $5.1 \mathrm{~cm}(\mathrm{SE}, 0.2 \mathrm{~cm})$. The average (between-subjects) velocity across participants was $3.2 \mathrm{~cm} / \mathrm{s}$ (SE, $0.5 \mathrm{~cm} / \mathrm{s}$ ). Statistical analysis (ANOVA) of average trajectory norm and velocity did not show any significant effects of congruency or side, or any interactions (all $P>0.05$ ). The standard deviations of the movement (all of the trials for all participants together) were $3.5 \mathrm{~cm}$ for the $X$ direction, $3.8 \mathrm{~cm}$ for the $Y$ direction, and $2.4 \mathrm{~cm}$ for the $Z$ direction (Fig. 2).

\section{Study 3 - CCE during synchronous and asynchronous hand movements}

\section{CCE analysis}

RTs across the eight experimental conditions ranged between $479 \mathrm{~ms}$ and $895 \mathrm{~ms}$. The CCE magnitude was found to be larger in the same-side conditions than in the different-side conditions, and also reflected synchrony. Statistical analysis of RTs (ANOvA) revealed a main effect of congruency $\left(F_{1,11}=46.19, P<0.0001\right)$, and a significant interaction between side and congruency $\left(F_{1,11}=26.82, P<0.001\right)$, confirming that CCEs were significantly larger in the same-side conditions than in the different-side conditions $\left(t_{1,11}=5.17, P<0.0001\right.$; Fig. S2).

Crucially, we also found a significant three-way interaction between synchrony, side, and congruency $\left(F_{1,11}=6.38, P<0.03\right)$. Neuman-Keuls post hoc analysis revealed that this was caused by a significant difference between the synchronous and asynchronous movement conditions, but only for the same-side congruent condition $(P<0.001)$, that was absent in the same-side incongruent condition $(P>0.35)$. We also found a significant difference between the synchronous and asynchronous movement conditions in the different-side incongruent conditions $(P<0.001)$ that was absent in the different-side congruent conditions $(P>0.05)$. In order to fully understand this synchrony-dependent effect, an additional analysis was performed, and revealed that CCEs in the same-side condition, as well as the difference between CCEs in the same-side condition and CCEs in the different-side condition, were significantly larger in the synchronous than in the asynchronous case $\left(t_{1,11}=2.59\right.$, $P<0.03 ; t_{1,11}=2.52, P<0.03$; Fig. 5 and Table 2). Statistical analysis of the error data revealed only a main effect of congruency $\left(F_{1,11}=13.71, P<0.005\right.$; Table 2$)$. A comparison between the 


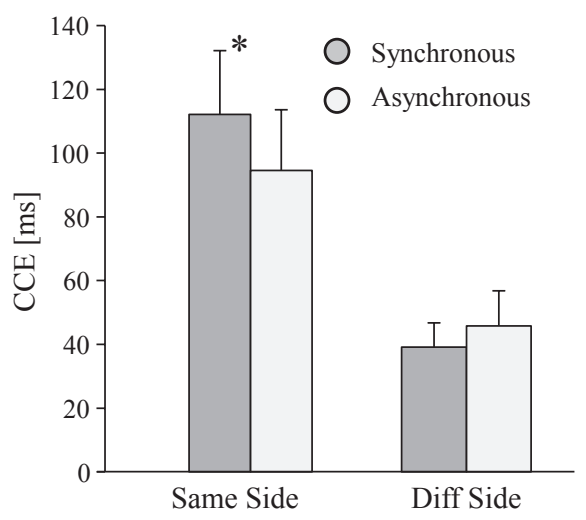

FIG. 5. Visuo-motor conflict decreases visuo-tactile integration (study 3). Mean CCEs (RTs) in the synchronous and asynchronous conditions, revealing an effect of synchrony that was observed only for same-side trials. Error bars show the standard error of the mean. See results for study 3 .

ANOVA on RTs and the ANOVA on accuracy revealed the same tendency for a common significant effect of congruency, suggesting that there were no speed-accuracy tradeoffs.

\section{Trajectory analysis}

In study 3, the average (between-subjects) trajectory norm was $13.3 \mathrm{~cm}(\mathrm{SE}, 0.7 \mathrm{~cm})$, and the average (between-subjects) velocity was $6.0 \mathrm{~cm} / \mathrm{s}(\mathrm{SE}, 0.6 \mathrm{~cm} / \mathrm{s})$. The ANOVA on trajectory norm did not show any significant effect of congruency, side, or synchrony, or any other interaction (all $P>0.05$ ). The differences in velocity and trajectory norm between study 2 and 3 might be mainly attributable to different groups of participants taking part in the studies.

Moreover, although the velocity in study 3 was double that in study 2, the movement can be still considered to be slow if compared with reaching movements. For instance, in a study using CCE and involving reaching movements, Brozzoli et al. (2009) found a velocity peak of approximately $120 \mathrm{~cm} / \mathrm{s}$, whereas the velocity in study 3 was $6 \mathrm{~cm} / \mathrm{s}$. This shows that, although the velocity was doubled between study 2 and study 3, both movements can be considered to be slow movements. The standard deviations of the movement (all of the trials for all participants together) for the $X$ direction were $4.4 \mathrm{~cm}$ in the synchronous condition and $4.6 \mathrm{~cm}$ in the asynchronous condition. For the $Y$ direction, the standard deviations were $4.1 \mathrm{~cm}$ in the synchronous condition and $4.2 \mathrm{~cm}$ in the

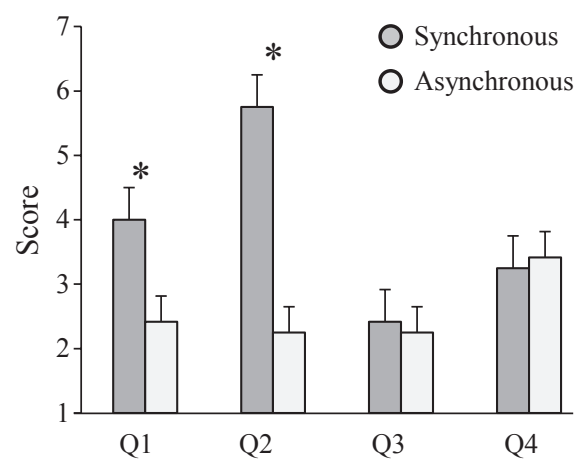

FIG. 6. Ownership and agency (study 3). Subjective ratings of agency and ownership, and control questions, are shown, based on a seven-point Likert scale (1, strongly disagree; 4 , not certain; 7 , strongly agree). asynchronous condition. For the $Z$ direction, the standard deviations were $4.1 \mathrm{~cm}$ in the synchronous condition and $4.3 \mathrm{~cm}$ in the asynchronous condition.

\section{Questionnaire analysis}

Questionnaire scores revealed that ratings in question Q1 regarding body ownership ('I felt as if the virtual hands were my hands') and ratings in question Q2 regarding agency ('I felt as if the movement of the virtual hands was my movement') were significantly larger in the synchronous condition than in the asynchronous condition $(\mathrm{Q} 1$, $z=2.66, P<0.01 ; \mathrm{Q} 2, z=3.059, P<0.01)$. For our two control questions (Q3 and Q4), there were no significant differences ( $z=0.91, P=0.36 ; z=0.07, P=0.94$; Fig. 6 ). These data show that body ownership and the sense of agency were manipulated with the present robotic setup (Table 1).

\section{Discussion}

The present study investigated the integration of visuo-tactile cues which had previously been studied almost exclusively in static conditions - under conditions in which participants performed repeated self-generated movements. In order to achieve visuo-tactile stimulation during bimanual hand movements, we modified an existing robotic training system for minimally invasive surgery and adapted it to previous experimental setups that were used to study visuo-tactile integration through the investigation of CCEs. First, the results show that a robotic-VR setup produces visuo-tactile integration results that are comparable to those obtained with real hands. Second, self-generated movements caused a reduction in CCEs. Third, delaying the visual feedback of self-generated movements resulted in reduced CCEs as compared with non-delayed feedback. Finally, delayed visual feedback caused a decrease in the sense of embodiment and agency with the virtual hands.

\section{Robotically mediated visuo-tactile integration}

The results of study 1 reveal that the CCEs in the current paradigm are comparable in terms of RT and accuracy to results obtained using typical paradigms (Spence et al., 2004a). Thus, we observed that RTs for incongruent trials were longer than those for congruent trials, and that CCEs were larger in the same-side than in the different-side conditions; that is, CCEs have a larger magnitude when the visual and tactile stimuli are closer to each other in space. This is consistent with several different experimental setups that have used visual distractors on rubber hands (Pavani et al., 2000), on real hands in different postures that were either crossed or uncrossed (Spence et al., 2004a), or on real hands separated by a horizontal distance of $35 \mathrm{~cm}$ (Shore et al., 2006).

Research in experimental psychology and neurophysiology has revealed that tools are powerful modulators of how the brain processes the body and its limbs, as well as peripersonal space, and that tools may be functionally integrated into a person's own body representation (Iriki et al., 1996; Maravita \& Iriki, 2004; Holmes et al., 2007b). The present data suggest that an extension of the body to incorporate the robot (which was not explicitly tested in the present experiments) is quite likely under the present experimental conditions, and that online CCEs may constitute an index that could quantify such 'robot-body' interactions (i.e. Maravita et al., 2002; Holmes et al., 2004, 2007a). Further work is needed to investigate such robotically mediated perceptual changes more directly. 


\section{The importance of movement in visuo-tactile integration}

The main goal of the present experiments was to assess how visuo-tactile integration is affected by self-generated actions. This question is especially pertinent, as sensory perception often takes place under highly dynamic conditions, and the perception of sensory cues must constantly be updated on the basis of the movements of the hands in peripersonal space (Klatzky et al., 2008). When we make hand movements, the brain needs to be able to distinguish between events related to our own actions and those linked to external objects or agents. Experimental evidence has provided support for the existence of an internal model that makes it possible to monitor self-generated actions and to recognise them as one's own (Wolpert et al., 1995; Blakemore et al., 1999; Frith, 2005; Bays et al., 2006), e.g. tactile stimuli occurring simultaneously with one's movement, which results in the attenuation of their perception (Angel \& Malenka, 1982; Milne et al., 1988; Collins et al., 1998; Bays et al., 2006; Juravle et al., 2010).

Despite this, earlier laboratory studies on visuo-tactile integration (i.e. when using CCE or related procedures) (Pavani et al., 2000; Spence et al., 2004a; Shore et al., 2006) did not employ interactive dynamic conditions (i.e. self-generated hand-arm movements), most likely because of the technical difficulties in controlling dynamic experimental setups. The present robotic setup allowed us to systematically test the effects of ongoing bimanual hand movements on visuo-tactile integration.

We report that: (i) classical effects of visuo-tactile integration (as measured via CCE magnitude) also occur during self-generated movements; and also that (ii) CCEs during self-generated movements differ from those when the hands are still. Concerning the first observation, we recorded larger CCEs in the same-side than in the different-side conditions across all trials (i.e. for static and movement conditions). This suggests that the present continuous movements did not interfere with the perceived co-localisation of visual and tactile cues for the horizontal plane; that is, those visual and tactile cues that are localised closer in space (same-side trials) interact more strongly than those that are further away (different-side trials), leading to larger CCEs in the former conditions. Same-side CCEs had a magnitude of $82 \mathrm{~ms}$, whereas different-side CCEs had a magnitude of only $44 \mathrm{~ms}$. These CCEs are of similar magnitude as those found in previous CCE studies in standard laboratory settings [Spence et al. (2004a), Study 1; Pavani et al. (2000), Experiment 1, rubber hands absent condition].

The critical new finding is the decrease in the CCE magnitude during self-generated movement. The absence of modulation of the CCE magnitude would have meant that visuo-tactile integration is not affected by self-generated movement, suggesting that predictive information regarding self-movements allows similar perceptual capacities and multisensory integration of those signals. Conversely, an increase in the CCE magnitude during self-generated action conditions might have resulted from attenuation of tactile signals, causing greater reliance on or salience of the visual distrators for localisation. The resulting decrease is compatible with the hypothesis that the brain uses different weightings of sensory information for the integration of visuo-tactile cues when moving; that is, there is a greater reliance on tactile signals. Interestingly, this effect is attributable to a selective difference during congruent trials (Fig. 4). Although, in the CCE literature, changes between conditions are typically attributable to differences in the incongruent trials (e.g. Spence et al., 2004a), a study adopting a reversed version of the cross-modal congruency task that we used in our experiments (i.e. judgements on the elevation of visual cues while ignoring tactile distractors) showed a CCE modulation attributable to larger differences in congruent than in incongruent trials (Walton \&
Spence, 2004). Furthermore, as the distractor can alter the responses in incongruent trials (by slowing them down), performance on congruent trials might show some degree of response facilitation (relative to a neutral baseline), owing to the spatial compatibility between the distractor and the stimulus (Marks, 2004; Spence et al., 2004b).

In study 2, the absence of a main effect of movement suggested no change in tactile sensitivity during dynamic conditions; accordingly, we argue that the effect arises from a reduction in visual saliency, i.e. lower cue reliability and therefore less interference. In a Bayesian perspective, lower reliability of vision can be modeled by assigning to the visual distractor a larger variance, which, according to the rules of optimal integration, leads to reduced weighting of vision. We suggest that this effect is driven by the difference in the congruent condition, as this requires precise spatial overlap (particularly to exert its facilitation role, as explained above). In other words, during hand movements, the visual distractor occurring in the congruent condition may be less perceived as occurring at exactly the same position as the tactile cue. The visual cue may, to some extent, be perceived at a different, more conflicting, spatial position. We therefore argue that movement decreases visual accuracy globally (i.e. not in a side-specific way), thereby reducing the up-down (congruent-incongruent) but not the left-right (side effect) difference assessed by the cross-modal congruency task. As this effect was only found in the movement condition, it could not have been found in previous CCE studies that focused on static paradigms.

As we did not record eye movements in the present experiments, we cannot rule out the possibility that attention-dependent mechanisms and fixation differences (e.g. eye movements) between the active and passive conditions may account for the different CCE magnitude found in study 2. However, the selective interference of movement in only the congruent trials, previous reports that visuotactile cues are attention-independent (Spence et al., 2004a,b) and our observation that congruency was modulated differently in sameside and different-side trials (in movement and static conditions) do not support this account. The data of study 3 - in which synchronous and asynchronous movement conditions were used - are also against such an account (see below). Moreover, recent studies have shown multisensory cues to be robust and to persist in perceptually demanding dual tasks in capturing exogenous spatial attention (Spence, 2010; Santangelo \& Spence, 2007, 2008). This further suggests that the CCE modulation found in study 2 is unlikely to be attributable to movement-related changes in attention or cognitive load.

A previous study has investigated the effects of visuo-tactile CCEs during movements of the upper limb (Brozzoli et al., 2009, 2010). However, several methodological differences make it difficult to directly compare these earlier data with our results. The present movements were continuous and bimanual movements that were not goaldirected. The studies by Brozzoli et al. required subjects to perform repeated goal-directed movements. Moreover, in the former goaldirected study, the visual distractors were not moving with and were not superimposed on the hand, but were placed statically on the target or goal. In the present study, visual distractors were moving, and they were superimposed on participants' hands during the CCE measurements. In addition, participants were not interacting with a robot as in the present experiments (haptic device), and Brozzoli et al. (2009) measured CCEs in three periods during the goal-direction action cycle, whereas we measured online CCEs during a continuous action cycle. Both studies revealed changes in visuo-tactile integration during movement. However, whereas the goal-directed movements were reported to lead to a progressive increase in CCE magnitude from the static (pre-motion) state to the action onset, and execution phase of a 
movement, we here observed a decrease in CCE magnitude during cyclic non-goal-directed movements.

More work along these lines is needed to compare different types of upper limb actions, including goal-directedness and its effects on visuo-tactile integration, especially because previous work has suggested that different behavioral tasks - such as perceptual judgements (e.g. drift measures in the rubber-hand illusion paradigm) or motor performance (e.g. reaching) - are often characterised by different rules governing multisensory integration and different visuotactile integration (Welch \& Warren, 1986; Ernst \& Bülthoff, 2004; Kammers et al., 2009, 2010; De Vignemont, 2010).

\section{Ownership, agency, and mislocalisation of touch}

The results of study 2 indicated that CCEs were lower in the movement condition than in the static condition. This suggests a lower reliance on visual information; however, as the stimulus movement was self-generated by the participant, predictive (efferent) information was also available. In study 3 , we also tested whether the predictive information arising from self-generated actions may have modulated the CCEs in the movement condition. This was done by introducing a delay between the participant's hand movement (as in study 2) and the seen hand movement (i.e. observed on the HMD).

We found a lower CCE for the same-side condition and a reduced difference between same-side and different-side conditions between the asynchronous and the synchronous conditions. This was driven by a smaller CCE for the asynchronous movements in the same-side condition. We suggest that the increased visual uncertainty in the asynchronous condition may have been caused by the incorrect predictive efferent information regarding the location of the hand (MonWilliams \& Tresilian, 1998; Van Beers et al., 2001). This finding shows that predictive efferent information is involved in multisensory integration. These results are in line with our prediction of a reweighting of the visual cues in visuo-tactile integration, and suggest that, in the asynchronous condition, participants perceived the visual distractors (placed on the moving virtual hands) as further away (and hence less relevant) with respect to the tactile stimuli. Indeed, previous studies (Spence et al., 2004a,b) have shown that the difference between the same-side and different-side CCEs is larger when the visual and tactile stimuli are perceived to be closer to each other in space. Thus, the greater the perceived proximity between the vibrotactile targets on the participants' hands and the visual distractors seen on the virtual hands, the greater the difference between the same-side and different-side CCEs (Pavani et al., 2000; Spence et al., 2004a,b; Aspell et al., 2009). Additionally, the reduced CCE in the asynchronous condition may also relate to the reduced sense of embodiment found in that condition. This may have further reduced the perceptual co-localisation of the visual and tactile cues, as the virtual hands no longer matched the participant's own hands (see below). Comparing study 2 and study 3, we suggest that decreased visual cue reliability resulting from movement hinders the up-down localisation, reducing the CCE magnitude at a global level, whereas the absence of predictive signals may cause localisation problems in a side-specific manner. We also note that, in study 3 , we did not find any specific effect in congruent trials (see study 2, Fig. 4), as the visual cue reliability was the same in both the synchronous and the asynchronous conditions (same motor signals).

Importantly, our results show side modulation of the CCE magnitude (significant interaction of the experimental factors side and congruency) across all three studies. This further suggests that participants have correct localisation of visuo-tactile cues across all of the conditions, and therefore that our frontal plane manipulation was effective. Velocity and trajectory analysis excluded the possibility that these selective CCE results depended on more basic movement differences across conditions. In the final experiment, we also tested whether the strength of body ownership and the sense of agency that our participants experienced in the robotic setup were modulated. As expected, our data show that ownership and agency during movement are preserved under synchronous conditions, and are significantly larger than in asynchronous conditions (Pavani et al., 2000; Austen et al., 2004; Aspell et al., 2009).

These data extend previous results showing that synchronous visuo-motor stimulation can induce illusory ownership of a virtual hand (Sanchez-Vives et al., 2010). Globally, our findings are in favor of a modulating role of self-generated movement signals in the integration of visuo-tactile cues. This suggests that current models of visuo-tactile integration need to be extended to account for multisensory integration under dynamic conditions. It will also be interesting to investigate in future studies whether and how the sense of body ownership changes during more complex bimanual movements and more complex interactions with objects (e.g. squeezing or picking up an object), and, importantly, whether these changes are reflected by online measures of body ownership, such as the CCE. Future detailed follow-up studies with the present setup may also investigate visuo-tactile integration during self-generated actions within a Bayesian framework (Roach et al., 2006; Körding et al., 2007; Wozny \& Shams, 2011).

\section{$V R$, robotics, and neuroscience}

The present investigation of visuo-tactile integration during self-generated movement was made possible by the use of VR and robotics technologies. VR involves the creation of rich multisensory experiences mediated through technology; the effectiveness of virtual environments has often been linked to the richness of sensory information and 'realness' of the experience (Held, 1992; Barfield \& Furness, 1995). In 1992, Sheridan proposed that the quantification of the participant's multisensory experience in these environments should involve a set of objective measures that quantify task performance and the subject's experience (Sheridan, 1992). Recently, the merging of VR with cognitive science has begun to fulfill this initial proposal (Sanchez-Vives \& Slater, 2005; Ijsselsteijn et al., 2006; Ehrsson, 2007; Lenggenhager et al., 2007; Slater et al., 2009; Slater et al., 2010; Bohil et al., 2011; Blanke, 2012). This study expands this earlier line of work by measuring the CCE in a VR setup to monitor changes in multisensory processing online, and the results suggest that the CCE is an effective metric with which to measure visuo-tactile integration in a virtual environment (Aspell et al., 2009). A recent functional magnetic resonance imaging study on the cortical multisensory aspects of self-consciousness has demonstrated that the further addition of robotics should powerfully enhance the capabilities of more classical VR setups (Ionta et al., 2011). Similarly, the present work shows that such robotic VR experiments are an important avenue to pursue in order to increase the control over a broad range of experimental designs involving many different dynamic and interactive (self-generated) multimodal sensory stimuli (Blanke \& Gassert, 2009).

\section{Outlook}

Collectively, the data from study 1 show that procedures from experimental psychology used to investigate the integration of visual and tactile stimuli can be transferred to setups using robotic technology. This suggests that employing CCE measurements during robotically 
mediated interactions may be an interesting and productive avenue to pursue, in order to quantify the so-called 'human factors' in robotically mediated perception and interactions (Holmes \& Spence, 2006). Thus far, human factors in robotics (mostly discussed in the field of minimally invasive robotic surgery) have most often been quantified by means of questionnaires or performance-based interviews (Prasad et al., 2002; Suematsu \& del Nido, 2004; Lee et al., 2005; Narazaki et al., 2006; Santos-Carreras et al., 2011). The latter techniques were tested during specific tasks (e.g. time required for task completion during a surgical procedure), but have neglected more basic and quantifiable mechanisms of human-machine interactions, such as multisensory, visuo-tactile perception (Prasad et al., 2002; Narazaki et al., 2006; Okamura, 2009). We propose that it should be studied whether and how such quantifiable visuo-tactile CCE effects relate to more classical analyses of human factors in current interfaces in medical robotics, and that they should, potentially, replace or extend the more standard analysis in this field (Das et al., 1999; Lee et al., 2005; Santos-Carreras et al., 2011). Our data also suggest that CCEs (and probably also other related measures of visuo-tactile integration) may function as online indices of the control of robotic tools and for the sense of body ownership and agency in robotic environments or the use of artificial limbs, and for robotic surgery [for related proposals, see Holmes \& Spence (2006)]. For example, the CCEs could be used as an index of body ownership and agency to test the importance of haptic feedback for tele-operated systems (Marescaux et al., 2001; Verner \& Okamura, 2007, 2009; Santos-Carreras et al., 2010). The magnitude of such online measures may reflect aspects of the surgeon's learning curve, and could be used to monitor performance during minimally invasive robotic surgery (Okamura, 2009). Concerning body ownership, our findings may also be of relevance for the design of prosthetic devices. Thus, recent research on amputees has used passive manipulations of body ownership (rubber-hand illusion), and has shown that visual capture of tactile sensations is associated with body ownership of a prosthetic hand (Ehrsson et al., 2008; Marasco et al., 2011). Our data reveal decreases in visual interference or dominance over tactile cues when able-bodied subjects are moving their hands. Similar mechanisms are probably also present in upper limb amputees when they are moving their prosthetic device. The present study describes different rules governing visuo-tactile integration during active conditions, suggesting an important role of predictive motor (and/or proprioceptive) signals, which need to be integrated into the design of future prosthetic and robotic devices with sensory feedback.

\section{Supporting Information}

Additional supporting information can be found in the online version of this article:

Fig. S1. Individual subject data for the congruency-movement interaction of study 2 .

Fig. S2. CCE side-effect of study 2.

\section{Acknowledgement}

We thank the anonymous reviewers for their helpful and constructive comments.

\section{Abbreviations}

CCE, cross-modal congruency effect; HMD, head-mounted display; RT, reaction time; SE, standard error; VR, virtual reality.

\section{References}

Angel, R.W. \& Malenka, R.C. (1982) Velocity-dependent suppression of cutaneous sensitivity during movement. Exp. Neurol., 77, 266-274.

Aspell, J.E., Lenggenhager, B., Blanke, O. \& Williams, M.A. (2009) Keeping in touch with one's self: multisensory mechanisms of self-consciousness. PLoS ONE, 4, e6488.

Austen, E.L., Soto-Faraco, S., Enns, J.T. \& Kingstone, A. (2004) Mislocalizations of touch to a fake hand. Cogn. Affect. Behav. Neurosci., 4, 170 181.

Ayav, A., Bresler, L., Brunaud, L. \& Boissel, P. (2004) Early results of oneyear robotic surgery using the Da Vinci system to perform advanced laparoscopic procedures. J. Gastrointest. Surg., 8, 720-726.

Barfield, W. \& Furness, T.A. (1995) Virtual Environments and Advanced Interface Design. Oxford University Press, USA

Bays, P.M., Flanagan, J.R. \& Wolpert, D.M. (2006) Attenuation of self-generated tactile sensations is predictive, not postdictive. PLoS Biol., 4, e28.

van Beers, R.J., Wolpert, D.M. \& Haggard, P. (2002) When feeling is more important than seeing in sensorimotor adaptation. Curr. Biol., 12, 834 837.

Blakemore, S.J., Frith, C.D. \& Wolpert, D.M. (1999) Spatio-temporal prediction modulates the perception of self-produced stimuli. J. Cogn. Neurosci., 11, 551-559.

Blanke, O. (2012) Multisensory brain mechanisms of bodily self-consciousness. Nat. Rev. Neurosci., 13, 556-571.

Blanke, O. \& Gassert, R. (2009) Total control in virtual reality and robotics. Front. Neurosci., 3, 110-111.

Bohil, C.J., Alicea, B. \& Biocca, F.A. (2011) Virtual reality in neuroscience research and therapy. Nat. Rev. Neurosci., 12, 752-762.

Brozzoli, C., Pavani, F., Urquizar, C., Cardinali, L. \& Farnè, A. (2009) Grasping actions remap peripersonal space. NeuroReport, 20, 913-917.

Brozzoli, C., Cardinali, L., Pavani, F. \& Farnè, A. (2010) Action-specific remapping of peripersonal space. Neuropsychologia, 48, 796-802.

Collins, D., Cameron, T., Gillard, D. \& Prochazka, A. (1998) Muscular sense is attenuated when humans move. J. Physiol., 508, 635-643.

Das, H., Zak, H., Johnson, J., Crouch, J. \& Frambach, D. (1999) Evaluation of a telerobotic system to assist surgeons in microsurgery. Comput. Aided Surg., 4, 15-25.

De Vignemont, F. (2010) Body schema and body image - pros and cons. Neuropsychologia, 48, 669-680.

Di Luca, M. (2010) New method to measure end-to-end delay of virtual reality. Presence-Teleop. Virt., 19, 569-584.

Ehrsson, H.H. (2007) The experimental induction of out-of-body experiences. Science, 317, 1048.

Ehrsson, H.H., Rosén, B., Stockselius, A., Ragnö, C., Köhler, P. \& Lundborg, G. (2008) Upper limb amputees can be induced to experience a rubber hand as their own. Brain, 131, 3443-3452.

Ernst, M. \& Bülthoff, H. (2004) Merging the senses into a robust percept. Trends Cogn. Sci., 8, 162-169.

Frith, C. (2005) The self in action:essons from delusions of control. Conscious. Cogn., 14, 752-770.

Haggard, P., Taylor-Clarke, M. \& Kennett, S. (2003) Tactile perception, cortical representation and the bodily self. Curr. Biol. 13, R170-R173.

Held, R. (1992) Telepresence. J. Acoust. Soc. Am., 92, 2458.

Holmes, N.P. \& Spence, C. (2006) Beyond the body schema: visual, prosthetic, and technological contributions to bodily perception and awareness. In Knoblich, G., Thornton, I., Grosjean, M. \& Shiffrar, M. (Eds), Human Body Perception from the Inside Out. Oxford University Press, USA, pp. 15-64.

Holmes, N.P., Calvert, G. \& Spence, C. (2004) Extending or projecting peripersonal space with tools? Multisensory interactions highlight only the distal and proximal ends of tools. Neurosci. Lett., 372, 62-67.

Holmes, N.P., Sanabria, D., Calvert, G.A. \& Spence, C. (2007a) Tool-use: capturing multisensory spatial attention or extending multisensory peripersonal space? Cortex, 43, 469-489.

Holmes, N.P., Calvert, G.A. \& Spence, C. (2007b) Tool use changes multisensory interactions in seconds: evidence from the crossmodal congruency task. Exp. Brain Res., 183, 465-476.

Ijsselsteijn, W.A., de Kort, Y.A.W. \& Haans, A. (2006) Is this my hand see before me? The rubber hand illusion in reality, virtual reality, and mixed reality. Presence-Teleop. Virt., 15, 455-464.

Ionta, S., Heydrich, L., Lenggenhager, B., Mouthon, M., Fornari, E., Chapuis, D., Gassert, R. \& Blanke, O. (2011) Multisensory mechanisms in temporo-parietal cortex support self-location and first-person perspective. Neuron, 70, 363-374. 
Iriki, A., Tanaka, M. \& Iwamura, Y. (1996) Coding of modified body schema during tool use by macaque postcentral neurones. NeuroReport, 7, 2325-2330.

Jacobs, R.A. (2002) What determines visual cue reliability? Trends Cogn. Sci., 6, 345-350.

Jeannerod, M. (2007) Being oneself. J. Physiol. (Paris), 101, 161-168.

Juravle, G., Deubel, H., Tan, H.Z. \& Spence, C. (2010) Changes in tactile sensitivity over the time-course of a goal-directed movement. Behav. Brain Res., 208, 391-401.

Kammers, M., De Vignemont, F., Verhagen, L. \& Dijkerman, H. (2009) The rubber hand illusion in action. Neuropsychologia, 47, 204-211.

Kammers, M.P.M., Mulder, J., de Vignemont, F. \& Dijkerman, H.C. (2010) The weight of representing the body: addressing the potentially indefinite number of body representations in healthy individuals. Exp. Brain Res., 204, 333-342.

Kenney, P.A., Wszolek, M.F., Gould, J.J., Libertino, J.A. \& Moinzadeh, A. (2009) Face, content, and construct validity of dV-trainer, a novel virtual reality simulator for robotic surgery. Urology, 73, 1288-1292.

Klatzky, R.L., MacWhinney, B. \& Behrmann, M. (2008) Embodiment, Egospace, and Action. Psychology Press, USA.

Körding, K.P., Beierholm, U., Ma, W.J., Quartz, S., Tenenbaum, J.B. \& Shams, L. (2007) Causal inference in multisensory perception. PLoS ONE, 2, e943.

Lanfranco, A.R., Castellanos, A.E., Desai, J.P. \& Meyers, W.C. (2004) Robotic surgery: a current perspective. Ann. Surg., 239, 14-21.

Lee, E., Rafiq, A., Merrell, R., Ackerman, R. \& Dennerlein, J. (2005) Ergonomics and human factors in endoscopic surgery: a comparison of manual vs telerobotic simulation systems. Surg. Endosc., 19, 1064-1070.

Lenggenhager, B., Tadi, T., Metzinger, T. \& Blanke, O. (2007) Video ergo sum: manipulating bodily self-consciousness. Science, 317, 1096-1099.

Lerner, M.A., Ayalew, M., Peine, W.J. \& Sundaram, C.P. (2010) Does training on a virtual reality robotic simulator improve performance on the da Vinci ${ }^{\circledR}$ surgical system? J. Endourol., 24, 467-472.

Loomis, J.M. \& Knapp, J.M. (2003) Visual Perception of Egocentric Distance in Real and Virtual Environments. Lawrence Erlbaum, Mahwah, NJ, pp. 21-46.

Marasco, P.D., Kim, K., Colgate, J.E., Peshkin, M.A. \& Kuiken, T.A. (2011) Robotic touch shifts perception of embodiment to a prosthesis in targeted reinnervation amputees. Brain, 134, 747-758.

Maravita, A. \& Iriki, A. (2004) Tools for the body (schema). Trends Cogn. Sci., 8, 79-86.

Maravita, A., Spence, C., Kennett, S. \& Driver, J. (2002) Tool-use changes multimodal spatial interactions between vision and touch in normal humans. Cognition, 83, B25-B34.

Marescaux, J., Leroy, J., Gagner, M., Rubino, F., Mutter, D., Vix, M., Butner, S.E. \& Smith, M.K. (2001) Transatlantic robot-assisted telesurgery. Nature, 413, 379-380.

Marks, L.E. (2004) Cross-modal interactions in speeded classification. In Calvert, G.A., Spence, C. \& Stein, B.E., (Eds), Handbook of Multisensory Processes. MIT press, USA, pp. 85-105.

Milne, R., Aniss, A., Kay, N. \& Gandevia, S. (1988) Reduction in perceived intensity of cutaneous stimuli during movement: a quantitative study. Exp. Brain Res., 70, 569-576.

Mon-Williams, M. \& Tresilian, J.R. (1998) A framework for considering the role of afference and efference in the control and perception of ocular position. Biol. Cybern., 79, 175-189.

Narazaki, K., Oleynikov, D. \& Stergiou, N. (2006) Robotic surgery training and performance. Surg. Endosc., 20, 96-103.

Okamura, A.M. (2009) Haptic feedback in robot-assisted minimally invasive surgery. Curr. Opin. Urol., 19, 102-107.

Pavani, F., Spence, C. \& Driver, J. (2000) Visual capture of touch: out-ofthe-body experiences with rubber gloves. Psychol. Sci., 11, 353-359.

Prasad, S.M., Maniar, H.S., Soper, N.J. \& Damiano, R.J. (2002) The effect of robotic assistance on learning curves for basic laparoscopic skills. Am. J. Surg., 183, 702-707.

Roach, N.W., Heron, J. \& McGraw, P.V. (2006) Resolving multisensory conflict: a strategy for balancing the costs and benefits of audio-visual integration. Proc. R. Soc, B Biol. Sci., 273, 2159-2168.

Salomon, R., Malach, R. \& Lamy, D. (2009) Involvement of the intrinsic/ default system in movement-related self recognition. PLOS ONE, 4, e7527.

Salomon, R., Szpiro-Grinberg, S. \& Lamy, D. (2011) Self-motion holds a special status in visual processing. PLOS ONE, 6, e24347.

Sanchez-Vives, M.V. \& Slater, M. (2005) From presence to consciousness through virtual reality. Nat. Rev. Neurosci., 6, 332-339.

Sanchez-Vives, M.V., Spanlang, B., Frisoli, A., Bergamasco, M. \& Slater, M. (2010) Virtual hand illusion induced by visuomotor correlations. PLoS ONE, 5, e10381.
Santangelo, V. \& Spence, C. (2007) Multisensory cues capture spatial attention regardless of perceptual load. J. Exp. Psychol. Hum. Percept. Perform., 33, 1311-1321.

Santangelo, V. \& Spence, C. (2008) Is the exogenous orienting of spatial attention truly automatic? Evidence from unimodal and multisensory studies. Conscious. Cogn. 17, 989-1015.

Santos-Carreras, L., Beira, R., Sengül, A., Gassert, R. \& Bleuler, H. (2010) Influence of force and torque feedback on operator performance in a VRbased suturing task. Appl. Bionics Biomech., 7, 217-230.

Santos-Carreras, L., Hagen, M., Gassert, R. \& Bleuler, H. (2011) Survey on surgical instrument handle design: ergonomics and acceptance. Surg. Innov., 19, 50-59.

Sheridan, T.B. (1992) Telerobotics, Automation, and Human Supervisory Control. MIT Press, USA.

Shore, D.I., Barnes, M.E. \& Spence, C. (2006) Temporal aspects of the visuotactile congruency effect. Neurosci. Lett., 392, 96-100.

Slater, M., Perez-Marcos, D., Ehrsson, H.H. \& Sanchez-Vives, M.V. (2009) Inducing illusory ownership of a virtual body. Front. Neurosci., 3, 214 220

Slater, M., Spanlang, B., Sanchez-Vives, M.V. \& Blanke, O. (2010) First person experience of body transfer in virtual reality. PLOS ONE, 5, e10564.

Spence, C. (2010) Crossmodal spatial attention. Ann. NY Acad. Sci., 1191, $182-200$.

Spence, C., Pavani, F. \& Driver, J. (2004a) Spatial constraints on visualtactile cross-modal distractor congruency effects. Cogn. Affect. Behav. Neurosci., 4, 148-169.

Spence, C., Pavani, F., Maravita, A. \& Holmes, N. (2004b) Multisensory contributions to the 3-D representation of visuotactile peripersonal space in humans: evidence from the crossmodal congruency task. J. Physiol. (Paris), 98, 171-189.

Suematsu, Y. \& del Nido, P.J. (2004) Robotic pediatric cardiac surgery: present and future perspectives. Am. J. Surg., 188, 98-103.

Triesch, J., Ballard, D.H. \& Jacobs, R.A. (2002) Fast temporal dynamics of visual cue integration. Perception (Lond.), 31, 421-434.

Tsakiris, M. \& Haggard, P. (2005) The rubber hand illusion revisited: visuotactile integration and self-attribution. J. Exp. Psychol. Hum. Percept. Perform., 31, 80-91.

Tsakiris, M., Haggard, P., Franck, N., Mainy, N. \& Sirigu, A. (2005) A specific role for efferent information in self-recognition. Cognition, 96, 215-231.

Tsakiris, M., Prabhu, G. \& Haggard, P. (2006) Having a body versus moving your body: how agency structures body-ownership. Conscious. Cogn. 15, 423-432.

Van Beers, R.J., Wolpert, D.M. \& Haggard, P. (2001) Sensorimotor integration compensates for visual localization errors during smooth pursuit eye movements. J. Neurophysiol., 85, 1914-1922.

Van Selst, M. \& Jolicoeur, P. (1994) A solution to the effect of sample size on outlier elimination. Q. J. Exp. Psychol., 47, 631-650.

Verner, L.N. \& Okamura, A.M. (2007) Effects of translational and gripping force feedback are decoupled in a 4-degree-of-freedom telemanipulator. In Proceeding WHC '07 Proceedings of the Second Joint EuroHaptics Conference and Symposium on Haptic Interfaces for Virtual Environment and Teleoperator Systems. IEEE Computer Society Washington, DC, USA, pp. 286-291.

Verner, L.N. \& Okamura, A.M. (2009) Force \& torque feedback vs force only feedback. In Proceeding WHC '09 Proceedings of the World Haptics 2009 - Third Joint EuroHaptics conference and Symposium on Haptic Interfaces for Virtual Environment and Teleoperator Systems. IEEE Computer Society Washington, DC, USA, pp. 406-410.

Walton, M. \& Spence, C. (2004) Cross-modal congruency and visual capture in a visual elevation-discrimination task. Exp. Brain Res., 154, 113120 .

Wei, L., Sourin, A. \& Sourina, O. (2008) Function-based visualization and haptic rendering in shared virtual spaces. Visual Comput., 24, 871880.

Welch, R. \& Warren, D. (1986) Intersensory interactions. In Boff, K.R. Kaufmann, L. \& Thomas, J.P. (Eds), Handbook of Perception and Human Performance. John Wiley, NY, pp. 1-36.

Wolpert, D.M., Ghahramani, Z. \& Jordan, M.I. (1995) An internal model for sensorimotor integration. Science, 269, 1880-1882.

Wozny, D.R. \& Shams, L. (2011) Computational characterization of visually induced auditory spatial adaptation. Front. Integrative Neurosci., 5, 75.

Zopf, R., Savage, G. \& Williams, M.A. (2010) Crossmodal congruency measures of lateral distance effects on the rubber hand illusion. Neuropsychologia, 48, 713-725. 